\title{
Application of orthogonal polynomials for analysis of input numerical data in the problems of mass transfer
}

\author{
P'yanylo Ya. D., P'yanylo H. M., Vasiunyk M. Ye. \\ Centre of mathematical modelling, Pidstrygach IAPMM NAS of Ukraine \\ 15 Dudayev Str., Lviv, Ukraine, 79005
}

(Received 1 September 2015)

\begin{abstract}
The investigations of the spectral methods are represented for solving applied tasks, in particular, the processing of digital information (problem of approximation, compression of information, filtration of signals, determination of nature of physical process which is modeled), also imposition of boundary conditions for the formulated problems of mathematical physics, etc. The essence of these methods consists in representation of known and required functions by orthogonal series in the chosen bases and construction of the algorithms for calculation of coefficients of these series (the generalized spectra). The methods of these calculation depend on the type of input information (analytic or discrete types). Approximation method for functions of two variables in domains of noncanonical form is suggested.
\end{abstract}

Keywords: orthogonal polynomials, use of spectral methods, information processing, generalized spectra

2000 MSC: 76B07, 76B99

UDC: 621.64 .029

\section{Introduction}

Spectral methods have been applied in many fields of mathematics and mechanics: digital information processing (approximation tasks, compression of data, signal filtering), solving problems of mathematical physics, and so on [1-5]. The essence of these methods is to present the known and the unknown functions by orthogonal series in the chosen bases and to develop algorithms for the calculation of coefficients of these series (generalized spectra). Methods of these calculations depend on the type of input data. Fourier, Haar and Walsh bases are the most used so far. The comparative analysis of spectral methods in these bases showed that not all requirements for the found solutions of formulated problems are met. Therefore, spectral methods in other orthogonal bases, for example Jacobi, Chebyshev-Laguerre, Hermite, and more are being developed [6-8].

Problems can be solved by application of spectral methods in case when functions included in the mathematical model of the physical process description are represented as convergent series on this basis. The theory of orthogonal series includes theorems which describe the conditions of functions representation by means of the corresponding orthogonal series.

In the case of applying of analytical methods to solving these problems, the parametric dependence of input data on the corresponding coordinates should be obtained. Methods for constructing parametric dependence in the case of one variable are well described in scientific literature. In the case of several variables, it is necessary to develop and investigate such methods.

The aim of this work is to develop algorithms for application of orthogonal polynomials to study mass transfer processes in complex systems, in particular, the movement of gas in pipelines and gas filtration in porous media. 


\section{Finding the generalized spectra based on quadrature formulas}

Let the polynomials $u_{n}(x)$ be orthogonal on the interval $[a, b]$ with the weight function $\omega(x)$, and the function $\varphi(x)$ is can be by a orthogonal series of these polynomials $[5,8]$.

$$
\varphi(x)=\sum_{n=0}^{\infty} \frac{\varphi_{n}}{r_{n}} u_{n}(x)
$$

Here $\omega(x)$ is the weight function, $r_{n}=\int_{a}^{b} \omega(x) u_{n}^{2}(x) d x$ is the normalizing multiplier,

$$
\varphi_{n}=\int_{a}^{b} \omega(x) u_{n}(x) \varphi(x) d x
$$

are coefficients of generalized spectrum.

Formula (2) for calculation of the values of the generalized spectrum can be used when analytical submission function, which is decomposed in the corresponding series, is known. Usually, in practice, function values in non-equidistant points and with low accuracy are known. In this case the quadrature formulas are used for calculating of the values of $\varphi_{n}$. If the values of function are known in the points that are placed within certain rules, optimal in some sense quadrature formula can be built.

It is known that $(N+1)$-st orthogonal polynomial has $N+1$ real root, which belongs to the interval of the orthogonality. Then, to calculate generalized spectra of $\varphi_{n}$, the optimal quadrature formula in $L_{2}$ is true

$$
\varphi_{n} \approx \sum_{j=0}^{N} \rho_{j}^{2} u_{n}\left(x_{j}\right) \varphi\left(x_{j}\right) .
$$

where $x_{j}, j=\overline{1, N+1}$ are the roots of the polynomial $u_{N+1}(x)$, that is $u_{N+1}\left(x_{j}\right)=0, \rho_{j}^{-2}=\sum_{i=0}^{N} u_{i}^{2}\left(x_{j}\right)$.

Among the spectral bases Jacobi bases polynomials $P_{n}^{(\alpha, \beta)}(x), x \in[-1,1]$ and Chebyshev-Laguerre ones $L_{n}^{\lambda}(t), t \in[0, \infty)$ deserve the attention, where $\alpha>-1, \beta>-1, \lambda>-1$ are free parameters, $n$ is order of the polynomial, in particular with place for submission [5]

$$
\begin{gathered}
\frac{1}{2}[\varphi(x+O)+\varphi(x-O)]=\sum_{n=0}^{\infty} \frac{\varphi_{n}}{r_{n}} P_{n}^{(\alpha, \beta)}(x), \\
f(t)=\sum_{n=0}^{\infty} \frac{f_{n} n !}{\Gamma(n+\lambda+1)} L_{n}^{\lambda}(t) .
\end{gathered}
$$

Here $\Gamma(x)$ is the Euler gamma-function.

Note, that the functions defined in the interval $[-1,1]$, will be denoted as $\varphi(x)$ and functions, defined in the interval $[0, \infty)$, as $f(t)$.

If the approximating functions $\varphi(x)$ and $f(t)$ are set analytically, then generalized parametric images of generalized spectra series (4) and (5) exist

$$
\begin{aligned}
\varphi_{n} & =\int_{-1}^{1} \omega(x) \varphi(x) P_{n}^{(\alpha, \beta)}(x) d x \\
f_{n} & =\int_{0}^{\infty} t^{\lambda} e^{-t} f(t) L_{n}^{\lambda}(t) d t, \quad n=\overline{0, \infty},
\end{aligned}
$$

where $\omega(x)=(1-x)^{\alpha}(1+x)^{\beta}$ is the weight function for Jacobi polynomials. 
For Jacobi polynomials $u_{n}(x)=P_{n}^{(\alpha, \beta)}(x)$ formula (2) and (3) will be as follows

$$
\begin{aligned}
& \varphi(x)=\omega(x) \sum_{n=0}^{\infty} \frac{\varphi_{n}}{r_{n}} P_{n}^{(\alpha, \beta)}(x), \\
& \varphi_{n} \approx W_{N} \sum_{i=1}^{N+1} \eta_{N, i} P_{n}^{(\alpha, \beta)}\left(x_{i}\right),
\end{aligned}
$$

where

$$
\begin{gathered}
\eta_{N, i}=\frac{\left(1-x_{i}\right)^{1-\alpha}\left(1+x_{i}\right)^{1-\beta}}{\left[P_{n}^{(\alpha, \beta)}\left(x_{i}\right)\right]^{2}} \varphi\left(x_{i}\right), \\
W_{N}=2^{\alpha+\beta-1} \frac{\Gamma(N+\alpha+2) \Gamma(N+\beta+2)}{(N+1) ! \Gamma(N+\alpha+\beta+2)} \frac{(2 N+\alpha+\beta+2)^{2}}{(N+\alpha+1)^{2}(N+\beta+1)^{2}}, \\
P_{N=1}^{(\alpha, \beta)}\left(x_{i}\right)=0, \quad i=\frac{1, N+1}{\Gamma(n+\alpha+1) \Gamma(n+\beta+1)} \\
r_{n}=2^{\alpha+\beta+1} \frac{\Gamma(n+\alpha+\beta+1)}{n !(2 n+\alpha+\beta+1) \Gamma(n+\alpha)}
\end{gathered}
$$

Regarding the series

$$
f(t)=t^{\lambda} \sum_{n=0}^{\infty} \frac{f_{n} n ! L_{n}^{\lambda}(t)}{\Gamma(n+\lambda+1)}
$$

formula (3) will look like

$$
f_{n}=\sum_{j=1}^{N} \frac{f\left(t_{j}\right) t_{j} L_{n}^{\lambda}\left(t_{j}\right)}{(N+1)^{2}\left[L_{N+1}^{\lambda}\left(t_{j}\right)\right]^{2}} .
$$

Here $t_{j}$ are the roots of $N$-th Chebyshev-Laguerre polynomial, that is $L_{N}^{\lambda}\left(t_{j}\right)=0, j=\overline{1, N}$.

Note, that depending on the characteristics of the problem, the image signal number (5) may be modified. For example,

$$
f(t)=\sum_{n=0}^{\infty} \frac{n ! \bar{f}_{n}}{\Gamma(n+\lambda+1)} L_{n}^{\lambda}(t)
$$

or

$$
f(t)=t^{\lambda} e^{-t} \sum_{n=0}^{\infty} \frac{n ! \hat{f}_{n}}{\Gamma(n+\lambda+1)} L_{n}^{\lambda}(t) .
$$

The quadrature formula is also modified according to these submissions (11).

Choice of the type of the approximation of the function $f(t)$ with number (10), (12) or (13) should be carried out on the basis of a priori information. If it is known that in the vicinity of zero, the approximated function has the power behavior with the index $\lambda$, then it is expedient to approximate it by the series (10) if, in addition, $f(t)$ decreases exponentially at boundlessness - by the series (13).

Chebyshev-Laguerre polynomials have the drawback that leads to the fact that for the large values of powers $n$ we have

$$
L_{n}^{\lambda}(t)=O\left(e^{t / 2} t^{-(2 \lambda+1) / 4} n^{(2 \lambda-1) / 4}\right) .
$$

This property of Chebyshev-Laguerre polynomials significantly narrows the class of problems which use orthogonal transformation (7), because computational difficulties arise when the appropriate series is summed up for large $t$. In practice, this problem is solved by the introduction of a scale multiplier. However, changing of the scale multiplier requires a resolution of the problem and leads to instability 
in the calculation of the original $f(t)$. To avoid this drawback Chebyshev-Laguerre transformation should be generalized as follows.

Let

$$
f_{n}=\int_{0}^{\infty} t^{\nu \lambda+\nu-1} e^{-\mu t^{\nu}} L_{n}^{\lambda}\left(\mu t^{\nu}\right) f(t) d t
$$

where $\mu>0,|\nu|<\infty, \nu \neq 0$.

Then $f(t)$ will be calculated by the formula

$$
f(t)=\sum_{n=0}^{\infty} \frac{n ! f_{n}}{\Gamma(n+\lambda+1)} L_{n}^{\lambda}\left(\mu t^{\nu}\right) .
$$

If the function is approximated by a number (10), we obtain the following quadrature formula to calculate the Laguerre spectrum

$$
f_{n}=\frac{1}{|\nu|} \mu^{-\lambda-1} \sum_{j=0}^{N} \frac{t_{j} L_{N}^{\lambda}\left(t_{j}\right) f\left[\left(t_{j} / \mu\right)^{1 / \nu}\right]}{(N+1)^{2}\left[L_{N+1}^{\lambda}\left(t_{j}\right)\right]^{2}} .
$$

Generalization of Laguerre-Fourier series entered in this manner allows to use all properties of Chebyshev-Laguerre polynomials during its research.

Note that, along with the use of Chebyshev-Laguerre generalized polynomials spectral decomposition can be applied in basis of Laguerre functions

$$
\varphi_{n}(t)=e^{-t / 2} L_{n}^{\lambda}(t), \quad \lambda>-1,
$$

which are orthogonal on the interval and retain all the basic properties of Chebyshev-Laguerre polynomials. If $n \rightarrow \infty$ and $t \rightarrow \infty$, then Laguerre functions $\varphi_{n}(t)$ direct to zero.

Let the function $f(t)$ is approximated by the partial sum of the series

$$
f(t)=t^{\lambda} e^{\gamma \tau} \sum_{n=0}^{\infty} \frac{f_{n}}{r_{n}} \varphi_{n}(t / h)
$$

where $\gamma(|\gamma|<\infty)$ and $h(0<h<\infty)$ are some constants, $r_{n}$ is the normalizing multiplier. Then for calculation of coefficients $f_{n}$ the next formulas are true

$$
f_{n}=\int_{0}^{\infty} e^{-\gamma h \tau} f(h \tau) \varphi_{n}(\tau) d \tau, \quad f_{n} \approx \sum_{m=0}^{N} \frac{\lambda_{m} e^{-\gamma h \lambda_{m}} f\left(h \lambda_{m}\right)}{\left[(N+2) \varphi_{N+2}\left(\lambda_{m}\right)\right]^{2}} \varphi_{n}\left(\lambda_{m}\right) .
$$

Here $\lambda_{m}$ is the root of the function $\varphi_{N+1}(\tau)=0$.

The fact that the roots of Laguerre functions $\varphi_{n}(t)$ coincide with the roots of Chebyshev-Laguerre polynomials in the interval of orthogonality implies from the formula (14).

In measuring devices information, typically, is recorded in equidistant points, but the roots of polynomials which are in quadrature formulas (9) are placed unevenly in the interval of orthogonality, in general. Therefore, in order to use ratio (9) to calculate the Fourier-Jacobi coefficients it is necessary for the values of function $\varphi(x)$ to be appointed in the roots of the corresponding Jacobi polynomial. Then, for processing of equidistant data the dependence that would give an opportunity to translate equidistant points $t_{i}$ into corresponding roots of Jacobi polynomials has to be found. As follows from the theory of orthogonal polynomials, the roots $x_{i}$ of an arbitrary Jacobi polynomial are placed unevenly; it complicates transformation of even uniform scale $t_{i}$ into uneven one $x_{i}$. 
If the interval of orthogonality $[-1,1]$ will be transferred into $[0, \pi]$ in accordance with the formula $x=\cos \Theta$, the roots of $\Theta_{i}=\arccos x_{i}$ will be placed in the interval $[0, \pi]$ almost uniformly to the accuracy of a few valuable numbers. This is the origin of the method for transfer of even scale $t_{i}$ into roots $x_{i}$ for the Jacobi's polynomial follows from here. Using linear replacement of $\Theta=a t+b$, we transfer values $t_{i}, i=\overline{1, N+1}$ into the roots of $\Theta_{i}$ so that $t_{1}$ goes to $\Theta_{1}$ and $t_{N+1}$ in $\Theta_{N+1}$. Then $\Theta=\frac{\Theta_{N+1}-\Theta_{1}}{t_{N+1}-t_{1}} t+\frac{\Theta_{1} t_{N+1}-\Theta_{N+1} t_{1}}{t_{N+1}-t_{1}}$.

Going in the last formula to variable $x$, we obtain

$$
x_{i}=\cos \left[\frac{\Theta_{N+1}-\Theta_{1}}{t_{N+1}-t_{1}} t_{1}+\frac{\Theta_{1} t_{N+1}-\Theta_{N+1} t_{1}}{t_{N+1}-t_{1}}\right], \quad i=\overline{1, N+1} .
$$

This formula makes possible the transfer of points $t_{i}$ of uniform scale into points $x_{i}$, which are the roots of the polynomial $P_{N+1}\left(x_{i}\right)=0$.

\section{Construction of generalized spectra for a random data setting}

Usually, in practice, points of signal measurements of $F(t)$ at the points $t_{i}, i=\overline{1, n}$ of the range $\left[t_{0}, t_{1}\right]$ are placed randomly. By definition of orthogonal Fourier-Jacobi spectra

$$
\begin{aligned}
\varphi_{n} & =\frac{1}{r_{n}} \int_{-1}^{1} \omega(x) \varphi(x) P_{n}^{(\alpha, \beta)}(x) d x= \\
& =\frac{2}{r_{n}\left(t_{1}-t_{0}\right)} \int_{t_{0}}^{t_{1}} \omega\left[\frac{2 t-\left(t_{1}-t_{0}\right)}{t_{1}-t_{0}}\right] F(t) P_{n}^{(\alpha, \beta)}\left[\frac{2 t-\left(t_{1}-t_{0}\right)}{t_{1}-t_{0}}\right] d t .
\end{aligned}
$$

Since the values of function $F(t)$ are known in the points $t_{i}$, then the integral in the last equation can be approximated in many ways, including the method of rectangular trapezium. Then

$$
\begin{gathered}
S_{i}=\frac{1}{2}\left(t_{i}-t_{i-1}\right)\left[F\left(t_{i}\right) \omega\left(x_{i}\right) P_{n}^{(\alpha, \beta)}\left(x_{i}\right)+F\left(t_{i-1}\right) \omega\left(x_{i-1}\right) P_{n}^{(\alpha, \beta)}\left(x_{i-1}\right)\right], \\
\varphi_{n}=\frac{2}{r_{n}\left(t_{1}-t_{0}\right)} \sum_{i=1}^{N} S_{i} .
\end{gathered}
$$

For $n=0$

$$
\varphi_{0}=\frac{1}{r_{0}\left(t_{1}-t_{0}\right)} \sum_{i=1}^{N}\left(t_{i}-t_{i-1}\right)\left[F\left(t_{i}\right) \omega\left(x_{i}\right)+F\left(t_{i-1}\right) \omega\left(x_{i-1}\right)\right] .
$$

\section{Smoothing (averaging) of the input data and the use of spectral decomposition for identifying of the nature of physical processes}

Let the value of function $F(t)$ be given at points $t_{i}, i=\overline{1, n}$ from an interval $[0, l]$, that are placed randomly. The averaging of the function on an interval $t \in[0, l]$ is set by a formula $F_{c}=\frac{1}{l} \int_{0}^{l} F(t) d t$.

If a function $F(t)$ is approximated by an orthogonal series

$$
F(t)=\sum_{n=0}^{\infty} \frac{F_{n}}{r_{n}} p_{n}(t)
$$

Mathematical Modeling and Computing, Vol. 2, No. 1, pp. 88-98 (2015) 
where $p_{n}(t)$ is the orthogonal polynomials on an interval $t \in[0, l], r_{n}$ is the rationing multiplier, then

$$
F_{n}=\frac{1}{l} \int_{0}^{l} F(t) p_{n}(t) d t .
$$

In the condition of single gravimetric function $F_{0}=\frac{1}{l} \int_{0}^{l} F(t) d t=F_{c}$, it means that a zero coefficient of orthogonal decomposition is the average value of the function in the interval of orthogonality. Thus zero and first coefficient of orthogonal decomposition are average value and dispersion (by standard deviation), accordingly.

A calculating experiment was based on the measured data of the pressure for the pipeline of $1.388 \mathrm{~m}$ internal diameter, which are submitted in the Table 1 , where $t$ is the time; $p$ is the pressure value.

Table 1. Values of the pressure in the stationary $\left(p_{\text {stat }}\right)$ and non-stationary $\left(p_{\text {non-stat }}\right)$ modes of gas flow for different values of the time $(t)$.

\begin{tabular}{|c|c|c|c|c|c|c|c|c|}
\hline$p_{\text {stat }}$ & $p_{\text {non-stat }}$ & $t$ & $p_{\text {stat }}$ & $p_{\text {non-stat }}$ & $t$ & $p_{\text {stat }}$ & $p_{\text {non-stat }}$ & $t$ \\
\hline 65.30 & 68.44 & 2750 & 65.80 & 63.32 & 2035 & 65.57 & 64.30 & 1211 \\
\hline 65.53 & 68.57 & 2761 & 64.88 & 63.52 & 2041 & 65.34 & 64.09 & 1245 \\
\hline 65.31 & 68.84 & 2785 & 64.88 & 63.72 & 2043 & 65.56 & 63.89 & 1480 \\
\hline 65.54 & 69.05 & 2795 & 65.41 & 63.93 & 2045 & 65.35 & 63.68 & 1491 \\
\hline 65.33 & 69.26 & 2806 & 65.70 & 64.14 & 2050 & 65.59 & 63.89 & 1521 \\
\hline 65.56 & 69.47 & 2810 & 65.25 & 64.35 & 2056 & 65.39 & 63.66 & 1525 \\
\hline 65.31 & 69.68 & 2850 & 65.25 & 64.56 & 2058 & 65.61 & 63.51 & 1530 \\
\hline 65.57 & 69.88 & 2861 & 65.46 & 64.78 & 2060 & 65.61 & 63.62 & 1532 \\
\hline 65.37 & 70.08 & 2885 & 65.83 & 64.93 & 2071 & 65.40 & 63.45 & 1535 \\
\hline 65.58 & 70.30 & 2890 & 65.40 & 65.19 & 2079 & 65.60 & 63.67 & 1735 \\
\hline
\end{tabular}

The zero and the first coefficients of orthogonal decomposition of the Legendre polynomials are calculated on the basis of the given values, that acquire values $\varphi_{0}=65.4535, \varphi_{1}=-0.0220$.

The spectral decomposition of the signals in the bases of the orthogonal polynomials can be used also for identifying of physical processes. This fact is confirmed by a numerical experiment; the results of that are given in Table 2 .

Table 2. Values of the first three coefficients of decomposition of the pressure in a series with the Legendre polynomials for stationary (stationary) and non-stationary (non-stationary) motion of gas.

\begin{tabular}{|c|c|c|c|c|c|c|}
\hline \multirow{2}{*}{$N$} & \multicolumn{3}{|c|}{ stationary } & \multicolumn{3}{c|}{ non-stationary } \\
\cline { 2 - 7 } & $F_{0}$ & $F_{1}$ & $F_{2}$ & $F_{0}$ & $F_{1}$ & $F_{2}$ \\
\hline 20 & 65.5304 & -0.110 & 3.8603 & 63.0763 & 0.6783 & 4.0807 \\
\hline 25 & 65.5250 & -0.071 & 2.7262 & 63.1008 & 0.4642 & 3.3597 \\
\hline 30 & 65.5259 & -0.063 & 2.1005 & 63.2187 & 0.0612 & 3.2366 \\
\hline 35 & 65.5117 & -0.011 & 1.3491 & 63.5404 & -0.7882 & 3.1628 \\
\hline 40 & 65.5407 & -0.084 & 1.6021 & 64.164 & -1.8828 & 3.021 \\
\hline 45 & 65.5555 & -0.089 & 7.5527 & 65.0998 & -2.5757 & 7.5264 \\
\hline 50 & 65.5488 & -0.054 & 5.3194 & 65.3663 & -2.7597 & 5.1843 \\
\hline 55 & 65.5294 & 0.0124 & 3.4739 & 65.7914 & -3.0852 & 3.3002 \\
\hline 60 & 65.5213 & 0.0334 & 2.6812 & 66.0704 & -3.3753 & 2.6087 \\
\hline 65 & 65.5172 & 0.0377 & 2.0642 & 66.4614 & -3.8549 & 2.2061 \\
\hline
\end{tabular}


The values of the amount of the first measurements of pressure from Table 1, on the basis of which are the basis for the Fourier-Legendre coefficients calculation, are given in the first column of Table 2. As it is shown above, a zero Fourier-Legendre coefficient defines the average arithmetic value of the measured value in a corresponding interval. Further, as in the case of Legendre polynomials, then, by definition,

$$
F_{1}=\frac{1}{l} \int_{0}^{l} F(t) p_{1}(t) d t=\frac{1}{l} \int_{0}^{l} F(t) t d t .
$$

Table 2 shows that as $N$ is the number of first measurements of pressure, by which the coefficients are calculated, then to obtain stable values for the expansion coefficients we have to take at least 50 measurements.

The last formula expresses the mathematical expectation of a random value. Analysis of the results given in Tables 1, 2 shows that this value can be used for identification of a character of the motion of gas in a pipeline. In particular, from the analysis of the results given in Table 2, follows, that if the first coefficient the module of Fourier-Jacobi is less than 1, then the process of gas motion is stationary, in another case - non-stationary.

\section{The use of spectral methods for the analysis of influence of errors of input data}

Not all the parameters can be measured by means of measurement devices during the study of physical processes of different nature. Many of them are calculated on the basis of the measured data according to certain formulas. The volume of gas in a pipeline, which is calculated on the basis of the measured values of the pressure on the ends of pipeline, belongs to such parameters. As the measured values contain different sort of errors, it results in errors in the parameters which are calculated. Different sort of errors of calculations errors - the loss of large numbers of operations with large and small numbers and so on occur quite often next to the errors of input data.

Let create a formula on the basis of which we will determine a gas volume in a pipeline. Formulas are implied

$$
\begin{gathered}
M=\rho V=\rho_{0} V_{0}, \quad p=\rho z R T \\
z=\frac{1}{1+f p}, \quad f=[24-0.21(T-273.15)] 0.98692327 \cdot 10^{-9} .
\end{gathered}
$$

Here $M, \rho, V$ are mass, density, volume of gas in the working environment, and $\rho_{0}, V_{0}$ are density and volume of gas at standard conditions. If you know the pressure at the beginning and at the end of the pipeline, then its average value is calculated as follows

$$
p_{c}=\frac{2}{3}\left(p_{0}+\frac{p_{k}^{2}}{p_{0}+p_{k}}\right) \text {. }
$$

Relative change in the loss of the volume of gas $\Delta q_{v}$ for the time during which the average value of the pressure changes $p_{c 0}$ to $p_{c 1}$ is calculated by the formula

$$
\Delta q_{v}=\frac{p_{c 1}-p_{c 0}}{\rho_{0} R T}\left[1+\left(p_{c 1}+p_{c 0}\right)\right] .
$$

In order to find the change of gas supply in the pipeline, it is necessary to multiply the value $\Delta q_{v}$ by the value of the storage capacity of the pipeline $V$, that is $\Delta Q_{0}=\Delta q_{v} V$.

Computing experiment concerning the input data processing was conducted based on the basis of the data presented in Table 1, 2. The results of calculations are shown in the form of figures.

In the case of the applied researches with discrete setting of input data it is necessary to develop methods of constructing parametric images of initial boundary conditions. The methods of finding 


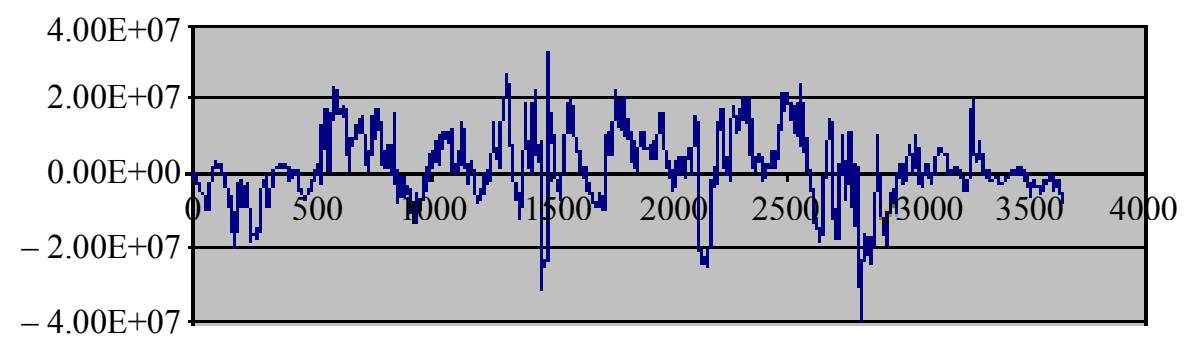

Fig. 1. Dependence of change of gas supply in a pipe $122 \mathrm{~km}$ long with a diameter of $1.388 \mathrm{~m}$, calculated on the basis of the measured data of input and output pressure in two hours interval without using a smoothing procedure.

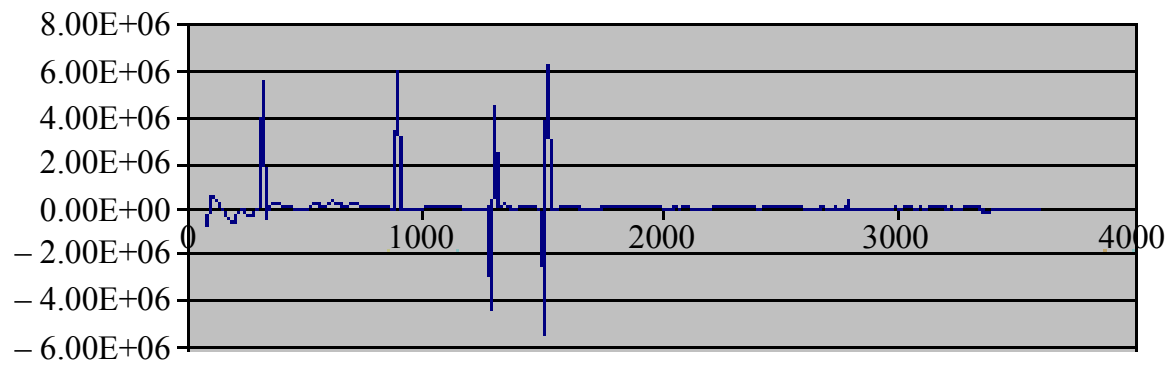

Fig. 2. Dependence of change of gas supply in a pipe $122 \mathrm{~km}$ long with a diameter of $1.388 \mathrm{~m}$, calculated on the basis of the processed measured data input and output pressure in twenty four hours interval.

solutions for the applied tasks must allow to control accuracy of calculations, exclude the rise of machine error and use a priori information about behavior of the unknown fields in certain areas.

Setting of parametric dependence for the case of two variables. We consider that discrete data are known in a rectangular area $x \in\left[x_{0}, x_{k}\right], y \in\left[y_{0}, y_{k}\right]$. We approximate the unknown function by an orthogonal series in two variables [9]

$$
p(x, y, 0)=p_{0}(\widetilde{x}, \widetilde{y})=\omega(\widetilde{x}) \omega(\widetilde{y}) \sum_{n, m=0}^{N, M} \frac{p_{n, m}}{r_{n} r_{m}} P_{n}^{(\alpha, \beta)}(\widetilde{x}) P_{m}^{(\alpha, \beta)}(\widetilde{y}) .
$$

Transition from coordinates $(x, y)$ to $(\widetilde{x}, \widetilde{y})$ is performed by formulas

$$
\widetilde{x}=\frac{2 x-x_{0}-x_{k}}{x_{k}-x_{0}}, \quad \widetilde{y}=\frac{2 y-y_{0}-y_{k}}{y_{k}-y_{0}},
$$

that is $\tilde{x} \in[-1,1], \tilde{y} \in[-1,1]$. Value of coefficients $p_{n, m}$ can be calculated using set values in the set points by quadrature formulas for one variable or by other methods.

1. The method of least squares is used for the minimization of the functional

$$
\delta=\sum_{j=1}^{J}\left[p_{0}\left(x_{j}^{0}, y_{j}^{0}\right)-p_{\vartheta}\left(x_{j}^{0}, y_{j}^{0}\right)\right]^{2},
$$

where $p_{\vartheta}\left(x_{j}^{0}, y_{j}^{0}\right)$ is the measured values of function at the points with coordinates $x_{j}^{0}, y_{j}^{0}$. Denote

$$
v_{n, m}(x, y)=\omega(x) \omega(y) \frac{1}{r_{n} r_{m}} P_{n}^{(\alpha, \beta)}\left(\frac{2 x-x_{0}-x_{k}}{x_{k}-x_{0}}\right) P_{m}^{(\alpha, \beta)}\left(\frac{2 y-y_{0}-y_{k}}{y_{k}-y_{0}}\right) .
$$


The minimum of the functional can be found from the condition $\frac{\partial \delta}{\partial p_{k, l}}=0(l, k=\overline{1, J})$. Then from the equation (15) we obtain

$$
\sum_{n, m=0}^{N, M} p_{n, m} \sum_{j=1}^{J} v_{n, m}\left(x_{j}^{0}, y_{j}^{0}\right) v_{k, l}\left(x_{j}^{0}, y_{j}^{0}\right)=\sum_{j=1}^{J} p_{f}\left(x_{j}^{0}, y_{j}^{0}\right) v_{k, l}\left(x_{j}^{0}, y_{j}^{0}\right) .
$$

If

$$
a_{n, m, l, k}=\sum_{j=1}^{J} v_{n, m}\left(x_{j}^{0}, y_{j}^{0}\right) v_{k, l}\left(x_{j}^{0}, y_{j}^{0}\right), \quad \varphi_{k, l}=\sum_{j=1}^{J} p_{f}\left(x_{j}^{0}, y_{j}^{0}\right) v_{k, l}\left(x_{j}^{0}, y_{j}^{0}\right)
$$

then

$$
\sum_{n, m=0}^{N, M} p_{n, m} a_{n, m, k, l}=\varphi_{k, l}
$$

The last formula can be written down as

$$
\sum_{s=1}^{S} p_{s} a_{s, r}=\varphi_{r}
$$

By providing variables $\left(x_{j}^{0}, y_{j}^{0}\right)$ relevant values and using known measured values of $p_{\vartheta}\left(x_{j}^{0}, y_{j}^{0}\right)$ in these points we calculate values $a_{s, r}$ and $\varphi_{r}$, and solve a system of equations (16) concerning the unknown coefficients $p_{s}$.

The system of linear equalizations (16) is poorly conditioned, and this fact does not allow to find the sufficient amount of coefficients $p_{s}$. In addition, the values of function usually are known with a low accuracy. This causes the occurrence of significant fatal error when during finding of the coefficients of orthogonal series.

2. Let us consider another way of calculation of coefficients $p_{n, m}$. Let us assume that the values of the pressure are known in points $\left(\widetilde{x}_{j}^{0}, \widetilde{y}_{j}^{0}\right)$, where $\widetilde{x}_{j}^{0}, \widetilde{y}_{j}^{0}$ are roots of polynomials of Jacobi $P_{N+1}^{(\alpha, \beta)}\left(\widetilde{x}_{j}^{0}\right)$ and $P_{M+1}^{(\alpha, \beta)}\left(\widetilde{y}_{j}^{0}\right)$. In such case it is possible to use a formula

$$
p_{n, m}=W_{N} W_{M} \sum_{i=1}^{N} \sum_{j=1}^{M} \eta_{N, i} \eta_{M, j} P_{n}^{(\alpha, \beta)}\left(\widetilde{x}_{j}^{0}\right) P_{m}^{(\alpha, \beta)}\left(\widetilde{y}_{j}^{0}\right) p_{f}\left(\widetilde{x}_{j}^{0}, \widetilde{y}_{j}^{0}\right)
$$

for the calculation of coefficients $p_{n, m}$.

3. The methods of construction of parametric dependence considered above can be used for rectangular areas. For the construction of self-reactance presentation of the unknown function in case of free-form area, which is placed in a rectangular area $x \in\left[x_{0}, x_{k}\right], y \in\left[y_{0}, y_{k}\right]$, it is possible to do following

Let $x \in\left[x_{0}, x_{k}\right]$, and the area is limited from below and from above by curves $y=f_{n}(x)$ and $y=f_{v}(x)$. In case of two variables parametric presentation of the generalized spectrum of FourierJacobi is such

$$
p_{n m}=\int_{x_{0}}^{x_{k}} \omega(x) P_{n}^{(\alpha, \beta)}(x) d x \int_{f_{n}(x)}^{f_{v}(x)} \omega(y) P_{m}^{(\alpha, \beta)}(y) p(x, y) d y .
$$

For the calculation of unknown coefficients it is possible to use an optimal in a class $L^{2}$ quadrature formula by turns after the variable of $y$ and $x$. The knots of quadrature formula for the variable $y$ will be placed on lines that pass through points $\left(x_{j}, f_{n}\left(x_{j}\right)\right)$ and $\left(x_{j}, f_{v}\left(x_{j}\right)\right)$. If knots for the variable $x$ are beyond the domain, then the value of the function proceeds with zeros. Let notice that in this case 
it is necessary to take into account the phenomenon of Gibbs on the border of range of definition for approximations of the unknown function.

\section{Conclusions}

1. With the increase of the length of the interval, the error of calculation will increase. Therefore, it is necessary to choose such a value of length of selection, in which the approximate value of zero coefficient $\varphi_{0}$ will be stable.

2. If the optimal value of the length of selection $l_{0}$ is found, then we calculate a value of $\varphi_{00}$, choosing a new start of counting. Obviously, in case of a steady process, $\varphi_{0}$ must be stable within the specified accuracy, hence $\varphi_{0}=\varphi_{00}$.

3. Similarly,

$$
\begin{aligned}
\varphi_{n}= & \frac{1}{r_{n}\left(t_{1}-t_{0}\right)} \sum_{i=1}^{N}\left(t_{i}-t_{i-1}\right) \times \\
& \times\left[F\left(t_{i}\right) \omega\left(x_{i}\right) P_{n}^{(\alpha, \beta)}\left(x_{i}\right)+F\left(t_{i-1}\right) \omega\left(x_{i-1}\right) P_{n}^{(\alpha, \beta)}\left(x_{i-1}\right)\right] .
\end{aligned}
$$

The approximated value of the signal is calculated with the values of coefficients $\varphi_{n}$ had been found as follows

$$
\varphi(x)=\sum_{n=0}^{\infty} \frac{\varphi_{n}}{r_{n}} P_{n}^{(\alpha, \beta)}(x) .
$$

The previous processing of data leads to stable results during the calculation of gas supply in a pipeline and change of gas supply. The analysis of input data (measured data) in relation to a character of motion shows, that the existing deviations in the calculations are related to the serious errors or defects in the process of recording of data.

[1] Lyalko V.I., Pjanylo Y.D. Using classical orthogonal polynomials in remote vegetation probing. Extras. NAS of Ukraine. No.2. P.42-46 (1997) (in Ukrainian).

[2] Pjanylo Y.D., Amerbaev V. M., Kireenko O. V., Kalynyak I. V. Calculation of Laguerre spectrum in the digital information processing. Space science and technology. Kyiv: Naukova Dumka. 5. P.45-48 (1990) (in Ukrainian).

[3] Hotra O., P'yanylo Ya. On Approach to the Modeling Process of the Flow of Blood in Vessels. Intelligent Information and Electronic Technology. 2008. IIET 2. P.103-119.

[4] Pjanylo Y., Hotra O. Methods of statistical processing of measured data in the base of Jacobi polynomials for environmental monitoring. Actual problems of economy. 10(112). P.221-230 (2010) (in Ukrainian).

[5] Suetin P. K. Classical orthogonal polynomials. Moscow: Nauka. 328 p. (1976) (in Russian).

[6] Pjanylo Y.D. Numerical analysis of generalized spectrum in the basis of Laguerre functions. Extras. NAS of Ukraine. No.12. P.49-53 (1994) (in Ukrainian).

[7] Pjanylo Y.D. Generalization of Laguerre integral transformation and its application. Proceedings of the Second Eng. Conf. with automatic control "Automatica-95". Lviv. 1. P.43-44 (1995) (in Ukrainian).

[8] Pjanylo Y. D. Projection-iterative methods for solving direct and inverse problems of transport. Lviv: Spline. 248 p. (2011) (in Ukrainian).

[9] Pjanylo Y., Vasyunyk M., Pjanylo G. Spectral methods of approximation of functions in the areas of noncanonical. II Scientific Conference "Computational methods and systems conversion information" October 4-5 (2012) (in Ukrainian). 


\title{
Застосування ортогональних многочленів для аналізу вхідних числових даних у задачах масопереносу
}

\author{
П'янило Я. Д., П'янило Г. М., Васюник М. Є. \\ Центр математичного моделювання ІППММ ім. Я. С. Підстригача НАН Украӥни \\ вул. Дж. Дудаєва, 15, 79005, Львів, Україна
}

Досліджено спектральні методи розв'язування прикладних задач, зокрема обробка цифрової інформації (задачі апроксимації, стиску інформації, фільтрації сигналів), визначення характеру фізичного процесу, який моделюється, та побудови крайових умов під час формулювання задач математичної фізики тощо. Суть цих методів полягає в поданні відомих і шуканих функцій ортогональними рядами у вибраних базисах і побудові алгоритмів для обчислення коефіцієнтів цих рядів (узагальнених спектрів). Методи їх обчислення залежать від способу задання вхідної інформації (в дискретному або аналітичному видах). Запропоновано спосіб апроксимації функцій двох змінних в областях неканонічної форми.

Ключові слова: ортогональні многочлени, використання спектральних методів, обробка інформачії, узагальнені спектри

2000 MSC: 76B07, 76B99

УДК: 621.64 .029 